\title{
DISTRIBUCIÓN ECOLÓGICA DE LOS OSTRÁCODOS DE LA CUENCA DE MALAMOCCO (LAGUNA DE VENECIA, NE DE ITALIA)
}

\author{
Francisco RUIZ ${ }^{\prime}$, María Luz GONZÁLEZ-REGALADO', \\ Laura MENEGAZZO ${ }^{2}$ y Giancarlo RAMPAZZO ${ }^{2}$
}

' Departamento de Geología, Universidad de Huelva, La Rábida, s/n, 21819-Palos de la Frontera (Huelva).

2 Dipartamento di Scienze Ambientali, Universitá Ca' Foscari de Venezia, Dorsoduro 2137, 30123-Venezia, Italia.

\begin{abstract}
Ruiz, F., González-Regalado, M.L., Menegazzo, L. y Rampazzo, G. 1999. Distribución ecológica de los ostrácodos de la cuenca de Malamocco (laguna de Venecia, NE de Italia). [Ecological distribution of ostracods in the Malamocco basin (Venice lagoon, northeastern Italy]. Revista Española de Paleontología, 14 (1), $59-65$. ISSN 0213-6937.
\end{abstract}

\begin{abstract}
The distribution of the Ostracoda in the central lagoon (Malamocco basin) of Venice is described. More than one hundred valves and caparaces were recognized belonging to twenty species. Three associations may be distinguished: (1) Cyprideis torosa association, located in the immediate vicinity of freshwater effluents, near the inner margin; (2) Xestoleberis communis association, present in areas of low energy; and (3) Pontocythere elongata association, with marine species introduced during the tidal flux and well represented near the entrance to the Malamocco Port. Ostracods have not been found in some environments with low water renewal, strong currents or low nutrient levels (macroalgae, phosphorus and nitrogen).
\end{abstract}

Keywords: Ostracoda, Ecology, Venice lagoon, Italy.

\section{RESUMEN}

Este trabajo describe la distribución de los ostrácodos en el sector central (cuenca de Malamocco) de la laguna de Venecia. Se identificaron más de un centenar de valvas y caparazones pertenecientes a 20 especies. Pueden diferenciarse 3 asociaciones de ostrácodos: (1) Asociación de Cyprideis torosa, bien representada en el margen lagunar interno sometido a aportes de aguas dulces; (2) Asociación de Xestoleberis communis, presente en medios con escaso gradiente energético; y (3) Asociación de Pontocythere elongata, formada por especies marinas introducidas por el flujo mareal en el área próxima a la entrada al Puerto de Malamocco. No se han encontrado ostrácodos en medios con escasa renovación de las aguas, fuerte hidrodinámica o bajos niveles de algas y otros alimentos de estos microorganismos.

Palabras clave : Ostrácodos, Ecología, laguna de V.enecia, Italia.

\section{INTRODUCCIÓN}

La laguna de Venecia es una amplia cuenca salobre $\left(550 \mathrm{~km}^{2}\right.$ de superficie) de escasa profundidad (más del $75 \%$ no supera el metro), localizada en la costa nororiental de Italia. Este espacio restringido está conectado con el mar Adriático mediante tres bocas de puerto (Fig. 1: Lido, Malamocco y Chioggia), con una profundidad comprendida entre 12 y $14 \mathrm{~m}$ y una anchura de 400 a $900 \mathrm{~m}$. Su origen se debe a los depósitos de los ríos Adige, Brenta, Bacchiglione y Piave (al $\mathrm{N}$ de la Fig. 1), con la creación de un lagoon litoral a finales de la glaciación Wurm (Gatto, 1980; Gatto y Carbognin, 1981).

Su actual fisiografía procede parcialmente de la erosión marina y de una acción antrópica especialmente intensa en los últimos 600 años. Durante los siglos XIV a XVII, los ríos que desembocaban en la laguna fueron desviados para evitar el hundimiento ocasionado por los aportes fluviales. En el siglo XVIII, numerosos espigones y muros fueron construidos en las islas-barrera externas para proteger la ciudad de Venecia y las poblaciones costeras, de la erosión marina. En 1922, comienza la producción industrial en el puerto de Marghera, con una 


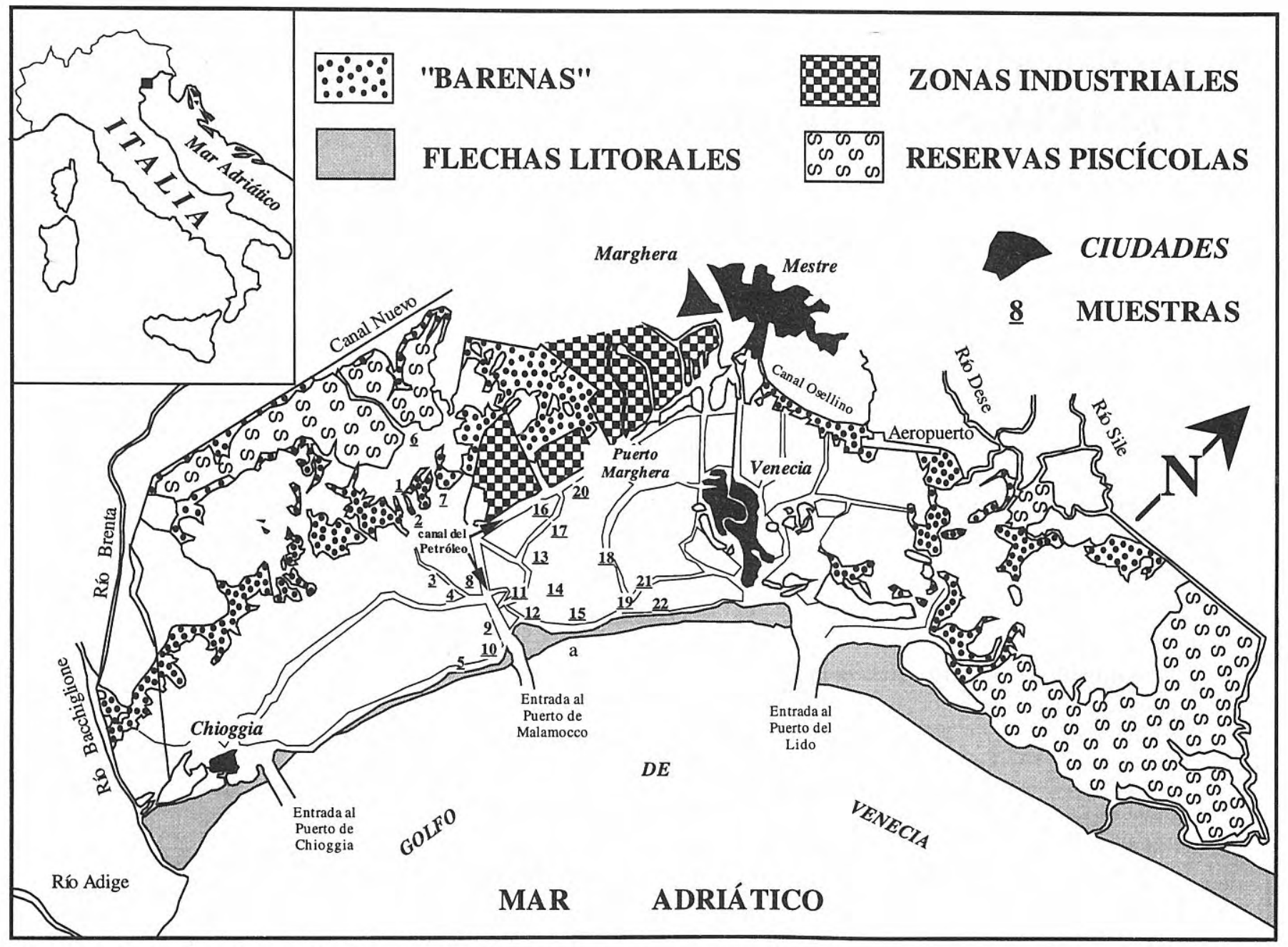

Figura 1. Mapa de la laguna de Venecia y localización de las muestras estudiadas.

superficie creciente de fábricas hasta 1970 (2.000 ha). Por último, entre 1950 y 1975, las masivas extracciones de aguas subterráneas y gas natural para uso industrial ocasionaron un hundimiento de 12-14 mm/año en el subsuelo lagunar (Avanzi et al., 1981).

Batimétricamente, la laguna presenta tres unidades morfológicas bien diferenciadas: (1) Las "barenas", término típicamente veneciano que alude a pequeñas islas emergidas durante la bajamar y caracterizadas por un borde externo elevado respecto a los bajos fondos circundantes y una parte interna deprimida (Romano, 1981); (2) las llanuras mareales, situadas entre los niveles de pleamar y bajamar, y (3) los canales y zonas pantanosas, siempre por debajo del nivel de bajamar.

Hidrológicamente, puede ser dividida en tres cuencas, cada una con conexión marina. De norte a sur, se diferencian las cuencas del Lido, Malamocco y Chioggia, con una superficie de 276, 162 y $111 \mathrm{~km}^{2}$, respectivamente. Estas tres cuencas están separadas por "vertientes" hidrodinámicas, con escasas velocidades de flujo $(<10$ cm/s). (Fig. 2, a) (Cavaleri, 1980).

La microfauna bentónica de la laguna de Venecia ha sido objeto de numerosos estudios, con especial énfasis en el análisis de las asociaciones de foraminíferos bentónicos, tanto actuales en algunos casos (Albani y Serandrei, 1982; Albani et al., 1987; Serandrei et al., 1989; Albani y Serandrei, 1990; Albani et al., 1991; Albani et al., 1995), como obtenidos en sondeos arqueológicos en otros (Albertotanza et al., 1977; Favero y Serandrei, 1980, 1981, 1983; Favero et al., 1995). En lo referido a ostrácodos, existe un fuerte contraste entre los diversos trabajos realizados en el mar Adriático (Masoli, 1969; Bonaduce et al., 1975; Breman, 1975) y la escasa información sobre los ostrácodos lagunares.

En este trabajo, se determinan los ostrácodos presentes en los sedimentos superficiales de la cuenca de Malamocco y se relacionan los datos obtenidos con los aportes de aguas dulces, las corrientes mareales, el tipo de sedimento y los niveles de nutrientes medidos en esta área.

\section{CARACTERIZACIÓN DE LA CUENCA DE MALAMOCCO}

\section{Aguas}

Los caracteres físico-químicos del agua presentan notables variaciones en el sector central de la laguna 


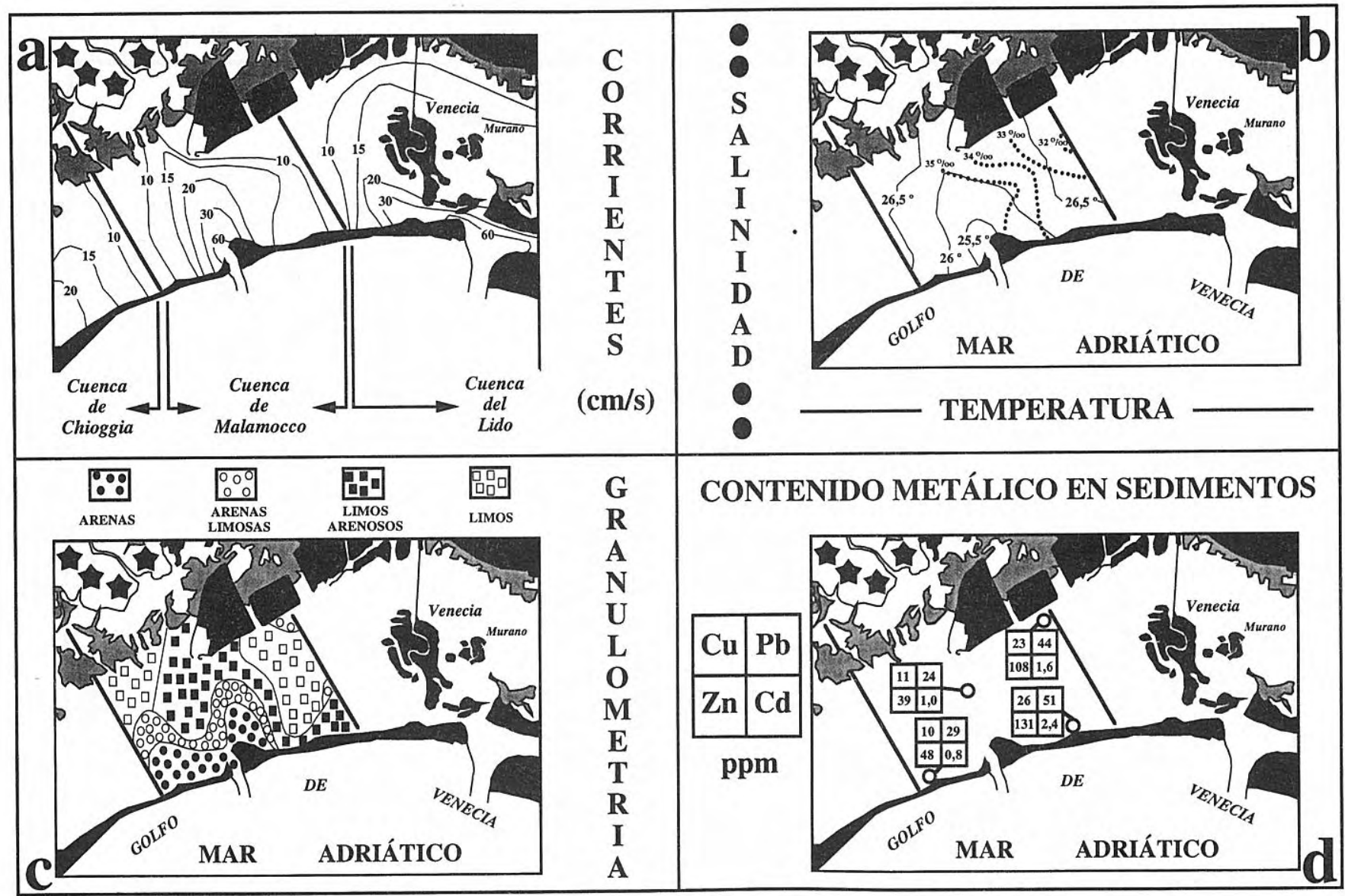

Figura 2. Caracterización de las aguas y sedimentos de la cuenca de Malamocco. a) Velocidad de corrientes (modificado de Cavaleri, 1980); b) Temperatura máxima en verano y salinidad (modificado de Marangon, 1996); c) Tamaño de grano (modificado de Barillari y Rosso, 1975; Barillari, 1981); d) Contenido en metales pesados (extraído de Donazzolo et al., 1984).

veneciana. La temperatura media anual es de $13-14{ }^{\circ} \mathrm{C}$, con máximos próximos a los $27^{\circ} \mathrm{C}$ en el verano (Fig. 2, b) y mínimos cercanos a los $0{ }^{\circ} \mathrm{C}$ durante el invierno en las áreas con mínimo flujo mareal (Albani y Serandrei, 1982). La salinidad se sitúa entre 33 y $35 \%$ en pleamar, disminuyendo levemente durante el reflujo mareal. Pueden producirse variaciones superiores al $10 \%$ cerca de la entrada de Malamocco, llegándose a los valores mínimos $(<5 \%$ ) cerca de algunos efluentes agrícolas e industriales (Marcello, 1967).

El $\mathrm{pH}$ permanece sobre 7,5 en otoño-invierno, experimentando importantes variaciones $(7,0$ a 8,5$)$ durante la primavera y el verano. El potencial redox suele oscilar entre +200 y $+400 \mathrm{mV}$; en algunos días de mayo y julio, un considerable aumento en la temperatura del agua, su escasa renovación y una enorme proliferación de macroalgas puede hacer bajar el Eh hasta valores comprendidos entre +70 y $-250 \mathrm{mV}$, con grandes mortandades de la macrofauna (Sfriso et al., 1988).

\section{Sedimentos}

El tamaño medio de grano disminuye hacia las zonas internas (Fig. 2, c). Las arenas son dominantes cerca de la entrada de Malamocco, donde las corrientes mareales son fuertes (más de 1,5 m/s) e impiden el depósito de los materiales más finos. En el resto, hay un dominio importante de la fracción pelítica, que puede sobrepasar el $70 \%$. Los sedimentos están formados principalmente por carbonatos, con dominio de dolomita sobre calcita y con la presencia de cuarzo, minerales de la arcilla y feldespatos como accesorios (Barillari y Rosso, 1975; Barillari, 1978; 1981; Hieke Merlin et al., 1979).

Los contenidos metálicos son importantes cerca de las zonas industriales localizadas en el puerto de Marghera, debido a los vertidos industriales y su distribución por los diversos canales mareales (Fig. 2, d). Esta zona es la principal fuente de $\mathrm{Cu}, \mathrm{Zn}$ y $\mathrm{Pb}$ hacia los sedimentos lagunares (Donazzolo et al., 1984), con focos menores en el puerto de Chioggia e industrias locales presentes en los márgenes.

\section{METODOLOGÍA}

La campaña de muestreo se realizó con el barco oceanográfico Umberto D'Ancona, perteneciente al Instituto de Biología del Mar (Consejo Nacional de Investigación-C.N.R.), y de una barca auxiliar del Laboratorio de Estudio de la Gran Masa del CNR durante los veranos de 1977 (muestras 19, 21 y 22) y 1978 (las 


\begin{tabular}{|c|c|c|c|c|c|c|c|c|c|c|c|c|c|c|}
\hline ESPECIES / MUESTRAS & 3 & 4 & 5 & 6 & 7 & 8 & 12 & 13 & 16 & 18 & 19 & 20 & 21 & 22 \\
\hline Aurila woodwardi & & & & & & 1 & 1 & & & & 3 & & & \\
\hline Callistocythere flavidofusca & & & & & & & 1 & & & & & & & 1 \\
\hline Cyprideis torosa (lisos) & & & & & & & 6 & & & & & & & \\
\hline Cyprideis torosa (nodosos) & & & & & & & & & 4 & & & & & \\
\hline Cyprideis torosa (punteado) & & & & 3 & & 1 & & & 36 & 1 & & 7 & & \\
\hline Cytheretta adriatica & & & 2 & & & & 1 & 1 & & & & & & \\
\hline Cytheroma variabilis & & & & & & & & & & & 1 & & & \\
\hline Hirschmania viridis & & & & & & & & & & 2 & & & 3 & \\
\hline Ilyocypris gibba & & & 1 & & & & & & & & & & & \\
\hline Leptocythere lagunae & & & & & & & & & & $\mathbf{1}$ & & & & \\
\hline Leptocythere ramosa & & & & & & & 1 & & & & & & & \\
\hline Leptocythere rara & & & & & & & 1 & & & & & & & \\
\hline Loxoconcha rubritincta & & & 1 & & & & 2 & & & & & & & \\
\hline Loxoconcha sp. & & & & & & & 1 & & & & & & & \\
\hline Neocytherideis fasciata & & & & & & & 2 & & & & & & & \\
\hline Phlyctocythere pellucida & & & & & & & & & & 2 & & & & \\
\hline Pontocythere turbida & & 1 & $\mathbf{5}$ & & 2 & 1 & 28 & & & & & & & \\
\hline Propontocypris pirifera & & & & & & & & & & & 1 & & & \\
\hline Semicytherura incongruens & & & & & & 1 & 2 & & & & & & & \\
\hline Semicytherura sulcata & & & & & & & 2 & & & & & & & \\
\hline Xestoleberis communis & 1 & & & & & & & & & 5 & 3 & 2 & 2 & \\
\hline Xestoleberis decipiens & & & & & & 1 & & & & & & & 3 & \\
\hline
\end{tabular}

Tabla 1. Distribución de los ostrácodos en las muestras analizadas.

restantes). Todas las muestras proceden de zonas de fondos medios y bajos, ya que los canales principales de la laguna (Fig. 1) están sometidos a un dragado continuo.

Se extrajeron $30 \mathrm{~cm}$ de testigo continuo mediante un testificador manual. De ellos, se separaron los 2-3 cm superficiales para el estudio microfaunístico. Una vez secados, una fracción $(25 \mathrm{~g})$ fue levigada por un tamiz de $63 \mu \mathrm{m}$ de diámetro de malla. El residuo fue secado en estufa a $70^{\circ} \mathrm{C}$ y posteriormente estudiado en gabinete. La determinación taxonómica se ha basado en los trabajos de Bonaduce et al. (1975), Breman (1975), Athersuch et al. (1989) y en diversos artículos del Stereo Atlas of Ostracod Shells (Doruk, 1974; Kilenyi y Whittaker, 1974; Athersuch y Whittaker, 1982).

\section{RESULTADOS Y DISCUSIÓN}

\section{Abundancia y diversidad}

Se han determinado taxonómicamente un total de 147 valvas y caparazones de ostrácodos pertenecientes a 20 especies. El número de especies por muestra se sitúa entre 0 y 12 , con los máximos valores en la muestra 12 , próxima a la entrada al canal del petroleo, con importante renovación del agua, pero protegida de la influencia directa de las corrientes mareales por una prolongación interna de las islas-barrera arenosas externas (Sfriso y Marcomini, 1996). Las formas más frecuentes son Cyprideis torosa (Jones), Pontocythere turbida (Muller) y Xestoleberis communis Muller, con una aparición esporádica de las restantes especies (Tabla 1). 


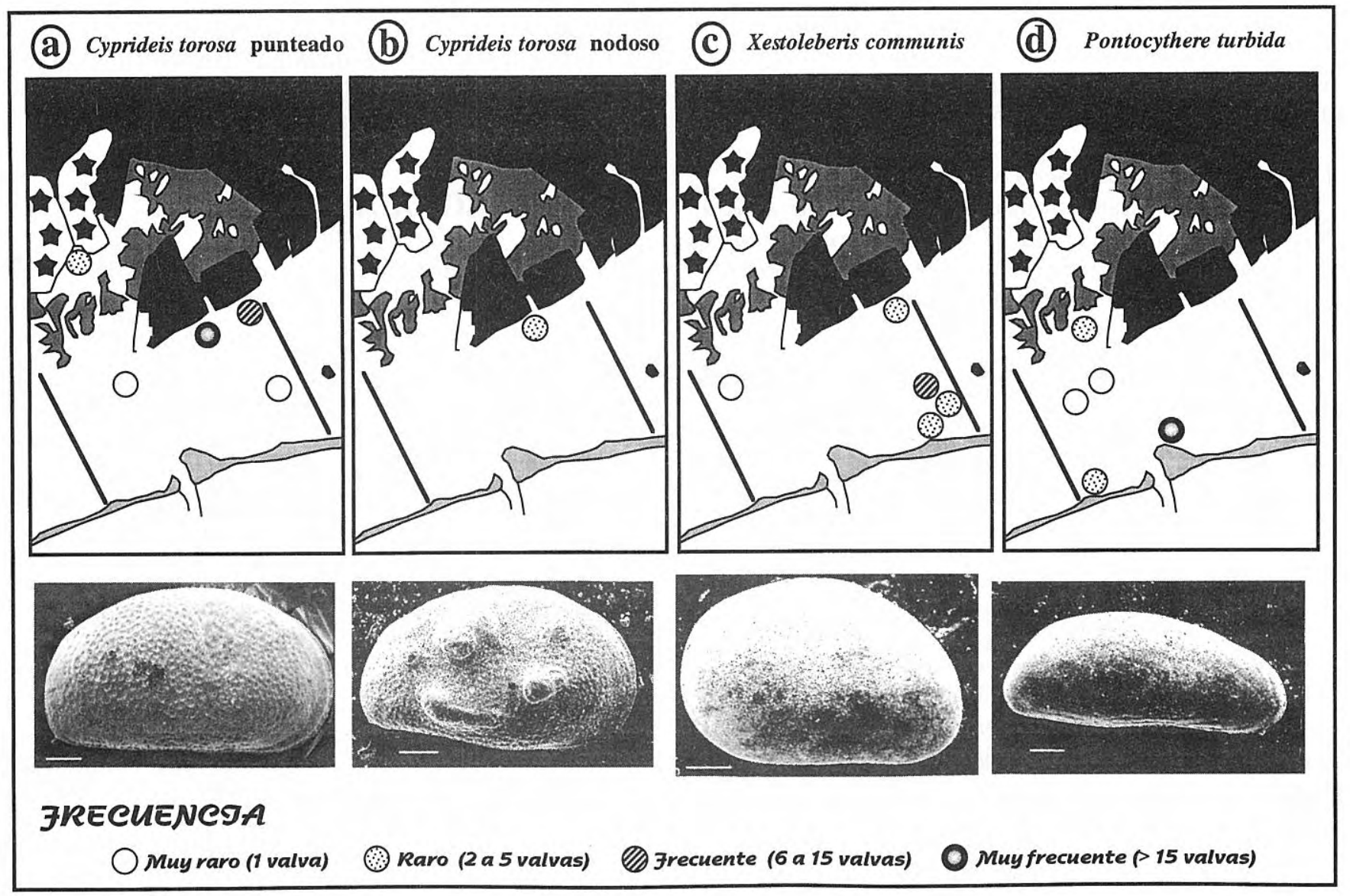

Figura 3. Distribución y abundancia de las principales especies de ostrácodos en la cuenca de Malamocco. Escala $=100 \mu \mathrm{m}$.

El número de individuos es escaso, con valores inferiores a 10 en la mayoría de las muestras. Sólo se supera esta cifra en las proximidades de la entrada al puerto de Malamocco (muestra 12) y en los bajos fondos próximos a la última zona industrial del puerto de Marghera. No se han observado ostrácodos en:

a) El sector más noroccidental de la cuenca (muestras 1 y 2), una zona formada por canales poco profundos, mal definidos y con una mínima renovación del agua durante la pleamar (Romano, 1981).

b) El principio del canal de conexión marina (muestras 9 a 11), debido a la inestabilidad provocada por las fuertes corrientes mareales $(>1,5 \mathrm{~m} / \mathrm{s})$ medidas en este sector (Sfriso et al., 1992). Estas desfavorables condiciones hidrodinámicas suelen provocar la desaparición de los ostrácodos en los canales principales de los ríos o los canales de entrada a estuarios o lagunas (Carbonel, 1980; Ruiz Muñoz et al., 1996).

c) La zona central de la cuenca (muestras 13,14 y 17), un sector con escasa biomasa algal $\left(<0,6 \mathrm{~kg} / \mathrm{m}^{2}\right)$ y bajas concentraciones de $\mathrm{P}$ y $\mathrm{N}$, algunas de las fuentes de alimentación de los ostrácodos (Sfriso et al., 1992; Sfriso et al., 1995).

\section{Asociaciones de ostrácodos}

Pueden diferenciarse 3 asociaciones de ostrácodos, con una distribución geográfica bien diferenciada:

\section{a) Asociación de Cyprideis torosa}

Esta especie aparece junto a esporádicos especímenes de Xestoleberis communis Brady en las muestras más próximas al margen lagunar interno, tanto en la proximidad del puerto de Marghera como en las zonas limítrofes con las reservas piscícolas, cerca de la desembocadura de pequeñas corrientes fluviales (Fig. 3, a y b). Es el sector con mayores fluctuaciones de salinidad, con bajas velocidades de las corrientes $(<10 \mathrm{~cm} / \mathrm{s})$ e importantes procesos de floculación de partículas de grano muy fino (dominio de limos). La importante contaminación detectada en los sedimentos de este área no parece afectar a esta especie, ya que la presencia de diversos estadios de crecimiento (mudas A-1 a A-6) junto a ejemplares adultos es indicativa de una biocenosis normal de medios poco energéticos (Whatley, 1988).

En esta área, esta especie presenta una ornamentación bien marcada (punteada o nodosa). Las formas punteadas son típicas de aguas mesohalinas o marinas normales $(>6$ $\%$ ), mientras que los ejemplares nodosos suelen aparecer en salinidades inferiores (Vesper, 1975; Carbonel et al., 1981; Aberkan et al., 1987). La presencia conjunta de ambas formas indica una estacionalidad en los aportes de aguas dulces, procedentes de algunos pequeños ríos y unos $2.000 \mathrm{~km}^{2}$ de agricultura extensiva (hasta un 
máximo de $650 \mathrm{~m}^{3} / \mathrm{s}$, según Donazzolo et al., 1984), así como de las industrias ubicadas en el puerto de Marghera, con unos efluentes (dulces) equivalentes a una ciudad de 40.000 habitantes (Sfriso y Marcomini, 1996).

\section{b) Asociación de Xestoleberis communis}

Incluye formas autóctonas, como Cyprideis torosa con caparazón punteado, Leptocythere lagunae Hartmann. Xestoleberis communis o Xestoleberis decipiens Muller, junto a escasos especímenes alóctonos procedentes de medios dulceacuícolas, como Ilyocypris gibba (Ramdhor), o eurihalinos [p.e., Callistocythere flavidofusca (Ruggieri), Phlyctocythere pellucida (Muller)]. Se ha observado en los sustratos limosos cercanos a las dos vertientes hidrodinámicas que configuran la cuenca de Malamocco, con velocidades mínimas de flujo (Fig. 3, c). Estas especies de Xestoleberis son formas fitales que suelen encontrarse al final del gradiente energético en diversos medios litorales europeos (Carbonel, 1980).

\section{c) Asociación de Pontocythere turbida}

Esta asociación está formada por especies marinas costeras alóctonas [Cytheretta adriatica Ruggieri, Loxoconcha rubricincta Ruggieri, Neocytherideis fasciata (Brady y Robertson), Semicytherura incongruens (Muller), Semicytherura sulcata (Muller)], típicas de los sustratos arenosos o limo-arenosos someros del mar Adriático (Bonaduce et al., 1975; Breman, 1975), que son introducidas durante los flujos mareales en el complejo lagunar y depositadas en las proximidades de los canales principales (Fig. 3, d). También incluye formas salobres autóctonas, como Leptocythere spp. o Cytherideis torosa con caparazón liso. Esta carencia de ornamentación es típica de este género en medios de salinidad marina normal (> $30 \%$ ), como lagunas litorales o estuarios (Vesper, 1975; Aberkan et al., 1987).

Se encuentra bien representada en las zonas próximas a la entrada de Malamocco, especialmente en áreas relativamente protegidas de las fuertes corrientes mareales (muestra 12). Estos sectores están expuestos a una importante renovación mareal, con un lavado de las fracciones finas del sedimento y el predominio de las fracciones arenosas. Los nutrientes son escasos, tanto en el agua como en los sedimentos (Sfriso et al., 1995).

\section{CONCLUSIONES}

La distribución de las asociaciones de ostrácodos en el sector central de la laguna veneciana está condicionada por la salinidad, hidrodinámica y nutrientes. La asociación de Cyprideis torosa es dominante en los sedimentos limosos próximos al margen lagunar interno, sometidos a importates fluctuaciones de la salinidad. La asociación de Xestoleberis communis abunda en fondos limo-arenosos con escasa velocidad de flujo, próximos a las vertientes hidrodinámicas que configuran la cuenca de Malamocco. La asociación de Pontocythere elongata está configurada por especies marinas alóctonas que son introducidas por el flujo mareal en las zonas adyacentes a la entrada al Puerto de Malamocco. No se han observado ostrácodos en áreas con mínima renovación mareal, fuerte hidrodinámica o bajos contenidos en nutrientes.

\section{AGRADECIMIENTOS}

Expresamos nuestro agradecimiento a los Dres. Luis Sánchez de Posada y R. C. Whatley por la revisión crítica del manuscrito, y a D. Cristobal Cantero, de los Servicios Generales de Investigación de la Universidad de Huelva, por la realización de las fotografías de M. E. B.

\section{BIBLIOGRAFÍA}

Aberkan, M., Cahuzac, B et Carbonel, P. 1987. Paléoenvironnements laguno-lacustres au cours du Pléistocene supérieur du littoral atlantique marocain. Bulletin de l'Institute des Sciences, 11, 117-123.

Albani, A. D. and Serandrei, R. 1982. A foraminiferal fauna from the lagoon of Venice, Italy. Journal of Foraminiferal Research, 12, 234-241.

Albani, A. D. e Serandrei, R. 1990. I Foraminiferi della laguna e del golfo di Venezia. Memorie di Scienze Geologiche, 48, 271-341.

Albani, A. D., Favero, V. e Serandrei, R. 1987. Situazioni di stress ambientale e microfaune a foraminiferi nella laguna a sud di Venezia. Rapporti e Studi dell'Istituto Veneto di Scienze, Lettere ed Arti, 9, 125-147.

Albani, A. D., Favero, V. and Serandrei, R. 1991.The distribution and ecological significance of recent Foraminifera in the lagoon south of Venice (Italy). Revista Española de Micropaleontología, 23 (2), 29-45.

Albani, A. D., Favero, V. e Serandrei, R. 1995. Condizioni paleoambientali nel sottosuolo di Venezia: la chiesa di S. Lorenzo. Rapporti e Studi dell'Istituto Veneto di Scienze, Lettere ed Arti, 12, 155-182.

Albertotanza, L., Serandrei, R. e Favero, V. 1977. I sedimenti olocenici della laguna di Venezia (bacino settentrionale). Bolletino della Società Geologica Italiana, 96, 243-269.

Athersuch, J. and Whittaker, J.E. 1982. On Propontocypris pirifera (G.W. Muller). Stereo Atlas of Ostracod Shells, 9 (13), 69-76.

Athersuch, J., Horne, D. J. and Whittaker, J. E. 1989. Marine and brackish water ostracods. Synopses of the British fauna (new series), 43, $343 \mathrm{pp}$.

Avanzi, C., Fossato, V., Gatto, P., Rabagliati, R., Rosa Salva, P. e Zitelli, A. 1981. Ripristino, conservazione ed uso dell'ecosistema lagunare Veneziano. Commune di Venezia, Italia, 199 pp.

Barillari, A. 1978. Prime notizie sulla distribuzione dei sedimenti superficiali nel barcino centrale della Laguna di Venezia. Atti Istituto Veneto di Scienze, 136, 125-134.

Barillari, A. 1981. Distribuzione dei sedimenti superficiali nel bacino meridionale della Laguna di Venezia. Atti Istituto Veneto di Scienze, 139, 87-109.

Barillari, A. e Rosso, A. 1975. Prime notizie sulla 
distribuzione dei sedimenti superficiali della Laguna Veneta. Memoir Biogeographica Adriatica, 9, 13-32.

Bonaduce, G., Ciampo, G. and Masoli, M. 1975. Distribution of Ostracoda in the Adriatic Sea. Publicazione della Stazione Zoologica di Napoli, 40, 1304.

Breman, E. 1975. The distribution of ostracodes in the bottom sediments of the Adriatic Sea. Tesis Doctoral Universidad Amsterdam, $125 \mathrm{pp}$.

Carbonel, P. 1980. Les ostracodes et leur intéret dans la définition des écosystèmes éstuariens et de plateforme continentale. Essais d'application à des domaines anciens. Mémoires de l'Institute Géologique du Bassin d'Aquitaine, 11, 1-350.

Carbonel, P., Legigan, P.H., Pujos, M., Saubadé, A. M., Bobier, C. et Jouirou, M. 1981. Evolution du Lac de Tunis. Un modèle de passage du milieu littoral à un milieu lagunaire. Actes Premier Congress National des Sciences de la Terre, Tunisie, 91-100.

Cavaleri L. 1980. Sediment transport in shallow lagoons. Nuovo Cimento, 3C 5, 527-540.

Donazzolo, R., Orio, A., Pavoni, B. and Perin, G. 1984. Heavy metals in sediments of the Venice Lagoon. Oceanologica Acta, 7, 25-32.

Doruk, N. 1974. On Semicytherura incongruens (G.W. Muller). Stereo Atlas of Ostracod Shells, 2 (18), 105112.

Favero, V. e Serandrei, R. 1980. Origine ed evoluzione della laguna di Venezia. Bacino meridionale. Lavori della Società Veneziana di Scienze Naturale, 5, 49-71.

Favero, V. e Serandrei, R. 1981. Evoluzione paleoambientale della laguna di Venezia nell'area archeologica tra Burano e Canale S. Felice. Lavori della Società Veneziana di Scienze Naturale, 6, 119-134.

Favero, V. e Serandrei, R. 1983. Oscillazioni del livello del mare ed evoluzione paleoambientale della laguna di Venezia nell'area compresa tra Torcello er il margine lagunare. Lavori della Società Veneziana di Scienze Naturale, 8, 83-102.

Favero, V., Hayvaert, F. e Serandrei, R. 1995. Motta S. Lorenzo: evoluzione dell'ambiente in un sito archeologico della laguna di Venezia. Rapporti e Studi dell'Istituto Veneto di Scienze, Lettere ed Arti, 12, 183218.

Gatto, P. 1980. Il sottosuolo del littorale veneziano. CNR Istituto Studio Dinamico Grandi Masse, Technical Report, Venezia, 108 pp.

Gatto, P. and Carbognin, L. 1981. The lagoon of Venice: Natural environmental rend and man-induced modification. Hydrological Science Bulletin, 26, 379391.

Hieke Merlin, O., Menegazzo Vitturi, L. e Smewato, G.
1979. Contributo alla conoscenza dei sedimenti superficiali della Laguna Veneta. Rapporti e Studi dell'Istituto Veneto di Scienze, Lettere ed Arti, 137, 3551.

Kilenyi, T.I. and Whittaker, J.E. 1974. On Cyprideis torosa (Jones). Stereo Atlas of Ostracod Shells, 2 (5), 21-32.

Marangón, L. 1996. Studio dei diversi ambienti lagunari tramite l'integrazione di osservazioni sui sedimenti e campionamenti delle acque con modelli idrodinamici diffusivi. Tesi di laurea (inédita), Universitá degli studi di Venezia Ca' Foscari, 120 pp.

Marcello, A. 1967. Per la conservazione della laguna di Venezia. Quaderni della Ricerca Scientifica del C. N. R., 38, 13-19.

Masoli, M. 1969. Distribution of the genus Semicytherura in the Northern Adriatic Sea betweeen Venice and Trieste. In: The Taxonomy, Morphology and Ecology of recent Ostracoda (Ed. J. Neale). Oliver \& Boyd, Edinburgh, 334-355.

Romano V. 1981. Aspetti sedimentologici della laguna di Venezia (Bacino meridionale) e del litorale antistante, desunti da analisis granumoletriche con sedigrafo 5000 $D$. Tesi di laurea (inédita), Universitá degli studi di Venezia Ca' Foscari, parte I, 104 pp.

Ruiz Muñoz, F., González-Regalado, M.L. y Morales, J.A. 1996. Distribución y ecología de los foraminíferos y ostrácodos actuales del estuario mesomareal del río Guadiana (SO España). Geobios, 29, 513-528.

Serandrei R., Albani, A.A. e Favero, V. 1989. Distribuzione dei foraminiferi recenti nella laguna a nord di Venezia. Bolletino della Società Geologica Italiana, 108, 279288.

Sfriso, A. and Marcomini, A. 1996. Italy-The Lagoon of Venice. Ecological Studies, 123, 339-368.

Sfriso, A., Pavoni, B., Marcomini, A. and Orio, A.A. 1988. Annual variations of nutrients in the Lagoon of Venice. Marine Pollution Bulletin, 19, 54-60.

Sfriso, A., Pavoni, B., Marcomini, A. and Orio, A.A. 1992. Macroalgae, nutrient cycles and pollutants in the Lagoon of Venice. Estuaries, 15, 517-528.

Sfriso, A., Pavoni, B. and Marcomini, A. 1995. Nutrient distributions in the surface sediment of the central lagoon of Venice. The Science of the Total Environment, 172, 21-35.

Vesper, B. 1975. To the problem of noding of Cyprideis torosa (Jones, 1850). Bulletin of American Paleontologyst, 65, 205-216.

Whatley, R. C. 1988. Population structure of ostracods: some general principles for the recognition of palaeoenvironments. In: Ostracoda in the Earth Sciences (Eds. P. De Deckker, J. P. Colin and J. P. Peypouquet). Elsevier, Amsterdam, 245-256. 